SECTION 4. Computer science, computer engineering and automation.

Aizhan Soltangalievna Tlebaldinova

Doctoral student of East Kazakstan State Technical University named after

D.Serikbayev, Kazakhstan

a tlebaldinova@mail.ru

Natalya Fedorovna Denissova

Candidate of physical and mathematical sciences

East Kazakhstan State Technical University named after D.Serikbayev, Kazakhstan

NDenisova@ektu.kz

\title{
THE DEVELOPMENT OF A MODEL OF A NUMBER PLATE RECOGNITION SYSTEM CONTROL IN REAL TIME
}

\begin{abstract}
The paper represents the model of a number plate recognition system control in real time which allows to optimally manage the load of a computer system. The developed model allows the flexibility to manage the process of license plate recognition using not only the parameters of the process of recognition but also a variety of scenarios that define the structure of the process.
\end{abstract}

Key words: number plate recognition system, processing of video streams, algorithms and technologies for license plate recognition.

\section{РАЗРАБОТКА МОДЕЛИ СИСТЕМЫ УПРАВЛЕНИЯ ПРОЦЕССОМ РАСПОЗНАВАНИЯ АВТОМОБИЛЬНЫХ НОМЕРОВ В РЕАЛЬНОМ ВРЕМЕНИ}

Аннотация: $B$ работе представлена модель адаптивной системь распознавания автомобильных номеров в режиме реального времени, которая позволяет оптимально управлять загрузкой вычислительной системы. Разработанная модель позволяет гибко управлять процессом распознавания автомобильных номеров, применяя не только параметры процесса распознавания, но и различные сценарии, определяюшие структуру проиесса.

Ключевые слова: система распознавания автомобильных номеров, обработка видеопотока, алгоритмы и технологии

Processing video streams in real-time is one of the most difficult areas of machine intellectualization. Intelligent systems of processing and analyzing video streams are more and more intensively used in various fields of human activity. They are most widely used when dealing with such tasks as securing various facilities, development of traffic management systems, etc. One of the areas of developing systems for processing and analyzing video streams is the development of systems for license plate recognition.

Today, the market offers quite a number of plate recognition systems, but there is no system produced in Kazakhstan. After analyzing the known plate recognition systems the following was revealed: firstly, many systems give unsatisfactory results for images of poor quality; secondly, they do not work with complex (diverse) images, since it is difficult to locate the required recognition area in them; and thirdly, they are all aimed at well-defined conditions (lighting, camera angle, brightness, etc.). Their high cost does not allow for mass introduction.

The peculiarity of developed system is that depending on the image quality and technical characteristics of the equipment the data processing is performed by automatically selecting a particular model and algorithms which in turn provides the system's rate of 
response. For this purpose the control system model for license plate recognition in real time was developed, it will be the basis for the proposed system.

The conceptual model of adaptive plate recognition system (fig.1) was constructed on the basis of the model proposed in [1, p.81]. Model in figure 1 allows to optimally manage the load of a computer system. The management of loading of the computing system is based on the statistical data counted by the results of single frames recognition.

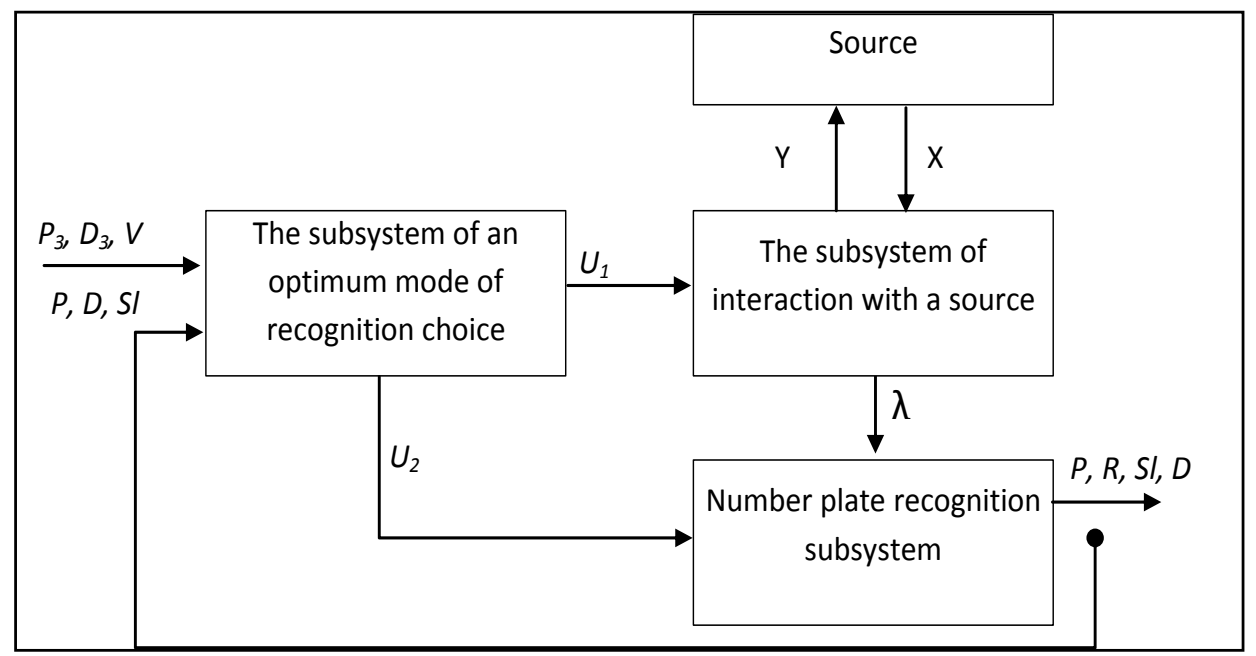

Figure 1 - Model of a number plate recognition system control in real time.

Input parameters of the model: set value $D_{3}$ - the reliability of license plate recognition, $V$ - vector of parameters.

$$
V=\left\{U_{10}, U_{20},\left[U_{1 \min }, U_{1 \max }\right],\left[U_{2 \min }, U_{2 \max }\right], K^{*}\right\},
$$

where $U_{10}, U_{20}$ - the initial values of parameters of the control recognition process, [ $U_{1 \mathrm{~min}}, U_{1}$ $\max ],\left[\begin{array}{lll}U_{2} \min , & U_{2} \max \end{array}\right]$ - ranges of change of the control parameters, $K^{*}-$ criterion of an optimality which is calculated by the formula (2).

$$
K^{*}=\min Z(P, D), P \geq P_{3}, D \geq D_{3}
$$

where $P_{3}$ and $P-$ a given and actual system performance as measured by the time of processing a single frame, $D_{3}$ and $D-$ a given and actual reliability of license plate recognition [2].

The system adaptation is divided into the following parts in this model:

- management of the video stream. Data coming to system through the device and the programs interacting with a source $U_{l}=\left\{R_{k}, L, F\right\}$, where $R_{k}$, - camera modes (resolution, frame frequency), $L$ - device parameters impact on the source, $F$ - the camera focusing);

- adapting the parameters of license recognition system. This part of the system selects recognition algorithms and processing methods $U_{2}=\{S, W\}$, where $S$ - the scenario of recognition determined by a vector of methods and algorithms, for example: $S=\left\{S_{\text {preproc }}\right.$, $\left.S_{\text {findNP, }}, S_{\text {segm }}, S_{O C R}, S_{\text {syntAN }}\right\}, W$ - vector of parameters of this scenario, for example: $W=\left\{W_{\text {preproc }}, W_{\text {findNP, }}, W_{\text {segm }}, W_{O C R}, W_{\text {syntAN }}\right\}$, thus $S_{\text {preproc }}$ - image pre-processing method, $W_{\text {preproc }}$ - image pre-processing method parameters, $S_{\text {findNP }}$ - search method of license plates, $W_{\text {findNP }}$ - search parameters of the method of license plates, $S_{\text {segm }}$ - method of image segmentation of license plate characters, $W_{\text {segm }}$ - parameters of the method of image segmentation of license plate characters, $S_{O C R}$ - method of character recognition, $W_{O C R}-$ parameters of the method of character recognition, $S_{\text {synt } A N}$ - parsing method of recognizing numbers, $W_{\text {syntAN }}$ - method parameters of recognized numbers parsing). Let us consider how 
this model works. Initially, the values of the control parameters are equal to the input parameters:

$$
U_{1}=U_{10}, U_{2}=U_{20}
$$

Interaction subsystem with a source can affect the source parameters $Y=\varphi_{1}(L)$. Received data are dependent on:

$$
X=\left\{x_{1}, x_{2}, \ldots, x_{k}\right\}, X=\varphi_{2}(Y)
$$

where $K$ - the number of signs.

Information density $\lambda$ is defined by the modes of interaction subsystem registration tools (video cameras). The subsystem receives the data frame by frame, so the flow is characterized by the amount of information $N$ in the frame which depends on the resolution of the camera, the frequency of transmitted snapshots $X^{*}=\left\{x_{1}^{*}, x_{2}^{*}, \ldots\right\}$ which in turn depends on the period between the previous frame of $\Delta t$.

$$
\lambda=X^{*}: \Delta t, N ; X^{*}=\varphi_{2}\left(U_{1}, X\right)
$$

The identification $R$ of car numbers (search numbers in the frame and recognizing characters in the each license plate) and the evaluation of recognition $S l$ and reliability $D$ are implemented in number plate recognition subsystem:

$$
R=S_{R}(W, \lambda), D=S_{D}(W, \lambda), S l=S_{S l}(W, \lambda)
$$

Since data is received in real time, the result of a license plate recognition is a function of time $R=f(t)$, as well as $S l=f_{1}(t), D=f_{2}(t)$. The calculation of mismatch between a given and actual evaluation of recognition reliability is performed in the subsystem of an optimum mode of recognition choice:

$$
\Delta D=D_{3}-D \text {. }
$$

After that, the optimal mode of operation of subsystems with the control parameters calculated for the specific case is set according to the criterion of $K^{*}$, as well as guided $\triangle D, S l, R$ and the vector of input parameters $V$ :

$$
U_{1}=F_{U 1}(V, \Delta D, S l, R), U_{2}=F_{U 2}(V, \Delta D, S l, R)
$$

The selection of the optimal mode is implemented by finding the minimum of the objective function $Z(P, D)$ for each moment of a regime change.

The developed model allows the flexibility of managing the license plate recognition process using not only the parameters of the recognition process, but also the variety of scenarios that define the structure of the process.

The greatest complexity in the implementation of the proposed model is to develop the number plate recognition subsystem which has to carry out automatic choice of this or that module depending on quality image.

The foundation of any ANPR system is recognition algorithms. Lotufo, Morgan and Johnson [2, pp.6/1-6/2] proposed an automatic license plate recognition system using optical character recognition methods. Fahmy [3, p.291] proposed a method for bidirectional associative memory neural network to read license plates. This method is suitable for a small number of models. Nijhuis, Ter Brugge, Helmholf J.P.W. Pluim, L. Spaanenburg, R.S. Venema and MAWestenberg [4, p.2233] proposed the use of fuzzy logic and neural networks for vehicle license plates. This method is used for the segmentation of fuzzy logic and discrete- time cellular neural networks for feature extraction . Choi [5, p.311] and Kim [6, p.260] proposed a method based on the use of the vertical edge of Hough transform to extract the license plate . E.R. Lee, P.K. And Kim H.J. Kim [7, p.307] used a neural network for extracting the color and pattern for character recognition . S.K. Kim, D.W. And Kim H.J. Kim $[8$, p.661] used a genetic algorithm for segmentation to extract the area with the license plate . Hontani [9, p.67] proposed a method of extracting characters without knowledge of their position and size of the image. Park et al [10, p.1475] developed a method to extract Korean license plates depending on the color plate. H.J. Kim, D.W. Kim, S.K. Kim, J.V. Lee, JKLee 
[11, p.215] proposed a method to extract the license plate area based segmentation of color images on the genetic distribution. Variety of approaches to finding the license plate shows how urgent this task.

There are many algorithms and technologies for license plate recognition and each of them has some advantages and disadvantages. Generally, these algorithms can be presented in the form of two groups of private algorithms (fig.2):

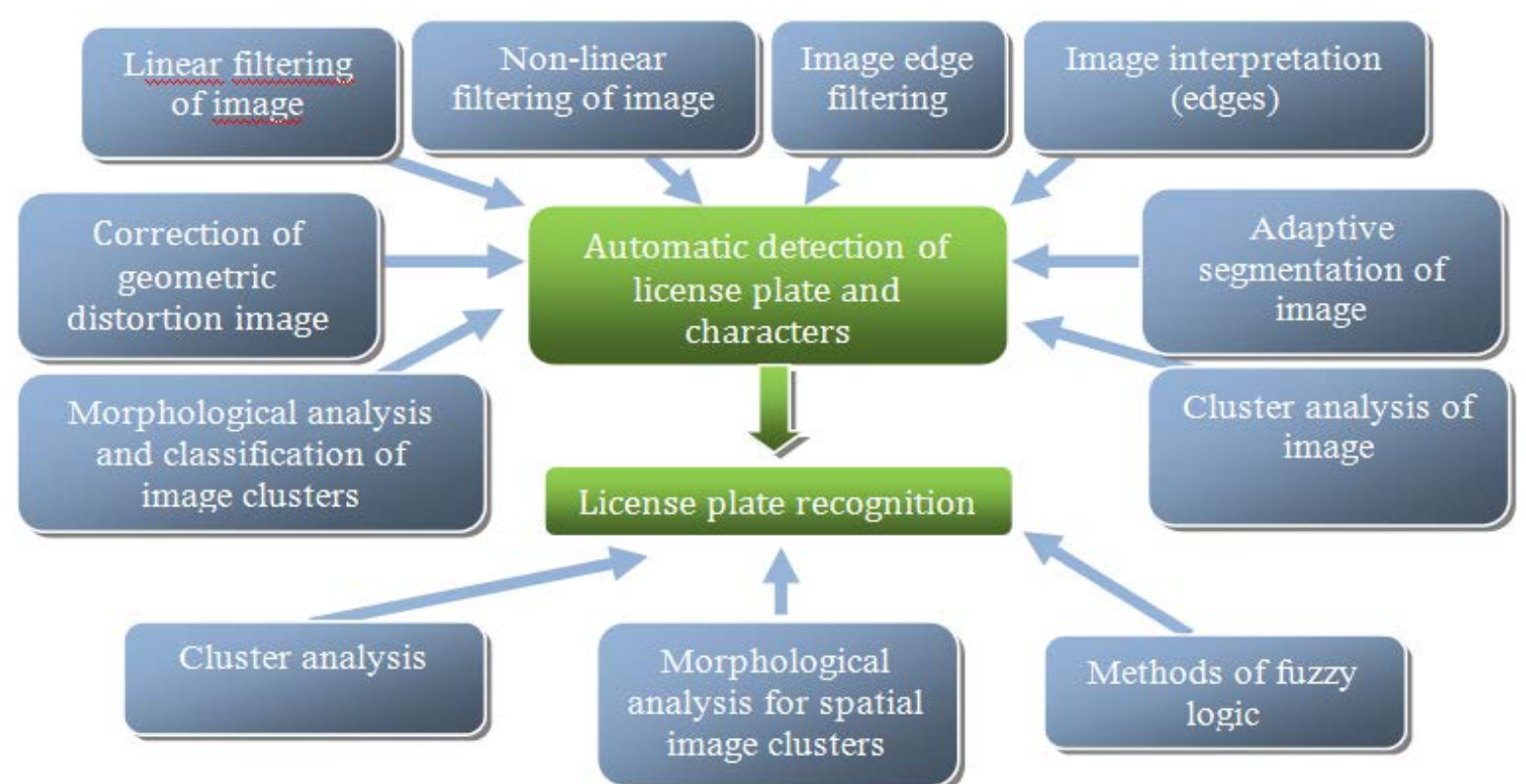

Figure 2 - The composition and the relationship of the algorithms used in automatic detection of the license plate within the contour of the vehicle and character recognition. vehicle;

1. Algorithms for automatic detection of license plate within the contour of the

2. Algorithms for license plate recognition.

The first group includes the following algorithms: algorithms for linear and non-linear filtering of images; algorithms for image edge filtering; algorithms for image edge interpretation; algorithm for adaptive segmentation of images; cluster analysis algorithm; algorithm for morphological analysis and classification of images clusters.

The second group includes the following algorithms: algorithms of morphological analysis for spatial images clusters; cluster analysis of characters; algorithm for fuzzy inference.

Thus, the choice of this or that algorithm significantly influences the performance of the entire model and the reliability of recognition. tasks:

Further development of the offered approach includes the solution of the following

- modernization of existing and development video streams recognition algorithms which will allow not only to get the results of recognition but also the values of their complexity and reliability;

- choice of strategy to set the optimum operating mode of the control plate recognition system;

- selection of hardware and software for the development of the system. 


\section{References:}

1. Kruchinin A.Yu., Aralbayev T.Z. Model of dynamic optimization of modes monitoring and borehole diagnosing // Izvestiya OrelSTU, series "Information Systems and Technologies". - 2006. - № 1 (2).p.81-85.

2. Lotufo R.A., Morgan A.D., and Johnson AS., 1990, Automatic Number-Plate Recognition, Proceedings of the IEE Colloquium on Image analysis for Transport Applications, V01.035, pp.6/1-6/6, February 16, 1990.

3. Fahmy M.M.M., 1994, Automatic Number-plate Recognition : Neural Network Approach, Proceedings of VNIS'94 Vehicle Navigation and Information System Conference, 31 Aug-2 Sept, pp.291-296, 1994.

4. Nijhuis J.A.G. , Brugge Ter M.H., Helmholt K.A., Pluim J.P.W., Spaanenburg L., Venema L., Westenberg M.A., 1995, Car License PlateRecognition with Neural Networks and Fuzzy Logic, IEEE International Conference on Neural Networks, pp.2232-2236, 1995.

5. Choi H.J., 1987, A Study on the Extraction and Recognition of a Car Number Plate by Image Processing, Journal of the Korea Institute of Telematics and Electronics, Vo1.24, pp. 309-315,1987.

6. Kim H.S., et al., 1991, Recognition of a Car Number Plate by a Neural Network, Proceedings of the Korea Information Science Society Fall Conference, Vol. 18, pp. 259262, 1991.

7. Lee E.R., P.K. Kim, and H.J. Kim, 1994, Automatic Recognition of a Car License Plate Using Color Image Processing, Proceedings of the International Conference on Image Processing, Lecture Notes in Computer Science, pp.307-314, 2005.

8. Kim S.K., Kim D.W., and Kim H.J., 1996, A Recognition of Vehicle License Plate Using a Genetic Algorithm Based Segmentation, Proceedings of 3rd IEEE International Conference on Image Processing, V01.2., pp.661-664, 1996.

9. Hontani H., and Koga T., (2001), Character extraction method without prior knowledge on size and information, Proceedings of the IEEE International Vehicle Electronics Conference (IVEC'01), pp. 67-72.

10. Park, S. H., Kim, K. I., Jung, K., and Kim, H. J., (1999), Locating car license plates using neural network, IEE Electronics Letters, vol.35, no. 17, pp. 1475-1477.

11. Kim H.J., Kim D.W., Kim S.K., Lee J.V., Lee J.K., 1997, Automatic Recognition of Car License Plates Using Color Image Processing, Engineering Design \& Automation, 3(2), pp.215-225, 1997. 